\title{
STRONG VARIABLE ULTRAVIOLET EMISSION FROM Y GEM: ACCRETION ACTIVITY IN AN ASYMPTOTIC GIANT BRANCH STAR WITH A BINARY COMPANION?
}

\author{
Raghvendra Sahai ${ }^{1}$, James D. Neill ${ }^{2}$, Armando Gil de Paz ${ }^{3}$, and Carmen Sánchez Contreras ${ }^{4}$ \\ ${ }^{1}$ Jet Propulsion Laboratory, MS 183-900, California Institute of Technology, Pasadena, CA 91109, USA \\ ${ }^{2}$ California Institute of Technology, 1200 E. California Blvd. MC278-17, Pasadena, CA 91125, USA \\ ${ }^{3}$ Dpto. de Astrofísica, Universidad Complutense de Madrid, E-28040 Madrid, Spain \\ ${ }^{4}$ Astrobiology Center (CSIC-INTA), ESAC campus, E-28691 Villanueva de la Canada, Madrid, Spain \\ Received 2011 July 15; accepted 2011 August 6; published 2011 September 28
}

\begin{abstract}
Binarity is believed to dramatically affect the history and geometry of mass loss in asymptotic giant branch (AGB) and post-AGB stars, but observational evidence of binarity is sorely lacking. As part of a project to look for hot binary companions to cool AGB stars using the Galaxy Evolution Explorer archive, we have discovered a late-M star, Y Gem, to be a source of strong and variable UV emission. Y Gem is a prime example of the success of our technique of UV imaging of AGB stars in order to search for binary companions. Y Gem's large and variable UV flux makes it one of the most prominent examples of a late-AGB star with a mass accreting binary companion. The UV emission is most likely due to emission associated with accretion activity and a disk around a main-sequence companion star. The physical mechanism generating the UV emission is extremely energetic, with an integrated luminosity of a few $\times L_{\odot}$ at its peak. We also find weak $\mathrm{CO} J=2-1$ emission from Y Gem with a very narrow line profile (FWHM of $3.4 \mathrm{~km} \mathrm{~s}^{-1}$ ). Such a narrow line is unlikely to arise in an outflow and is consistent with emission from an orbiting, molecular reservoir of radius $300 \mathrm{AU}$. Y Gem may be the progenitor of the class of post-AGB stars which are binaries and possess disks but no outflows.
\end{abstract}

Key words: accretion, accretion disks - binaries: general - circumstellar matter - stars: AGB and post-AGB stars: individual (Y Gem, Mira) - stars: mass-loss

\section{INTRODUCTION}

Binarity, very common among pre-main-sequence stars (e.g., Bodenheimer et al. 2000), is widely believed to dramatically affect the history and geometry of mass loss in late-AGB and post-AGB stars (e.g., Balick \& Frank 2002). The evolutionary transition from the asymptotic giant branch (AGB) to the planetary nebula (PN) phase is accompanied by significant changes in the morphology of these objects- the roughly round circumstellar mass-loss envelopes of AGB stars evolve into pre-planetary-nebulae (PPNs) and PNs with a dazzling variety of shapes and intriguing aspherical symmetries (e.g., Schwarz et al. 1992; Sahai \& Trauger 1998; Sahai et al. 2007, 2011). Binarity provides a source of angular momentum, as well as a preferred axis to a stellar system. Critical reviews (e.g., Soker 1998) of the properties of bipolar PNs lead to the conclusion that binary models can explain all these properties, whereas single-star models have many difficulties.

However, direct observational evidence for binarity in dying intermediate-mass stars is difficult to obtain. AGB stars are very luminous $\left(\sim 10^{3}-10^{4} L_{\odot}\right)$ and surrounded by dusty envelopes, whereas nearby stellar companions are generally likely to be significantly less luminous main-sequence stars or white dwarfs. Indirect techniques such as radial-velocity (RV) measurements or photometric-variability (PV) measurements cannot be used for AGB stars since their strong periodic or irregular variability intrinsic to their pulsating atmospheres masks the corresponding variability due to a companion. PV measurements of the central stars of PNs (CSPNs) have resulted in detecting a total of about 40 binaries (e.g., Bond 2000; Miszalski et al. 2011), implying a $10 \%-15 \%$ fraction of detectable close binaries among randomly selected PNs. RV measurements of CSPNs and post-AGB (pAGB) stars with prominent outflows (i.e., PPNs) have had very limited success (e.g., de Marco 2009; Hrivnak et al. 2011) because of the unknown contribution from stellar variability. Only for disk-prominent pAGB stars (dpAGB; Sahai et al. 2011), have RV techniques reached a $100 \%$ success rate in detecting binarity (e.g., Van Winckel et al. 2009).

Thus, new techniques to search for the presence of binary companions in AGB stars are of fundamental importance. Currently, ultraviolet photometry appears to be the most promising technique for being able to discover a substantial number of binary AGB stars. An as yet untested method which could become important is to look for X-rays from AGB companions which have been spun up via accretion (Soker \& Kastner 2002). Most AGB stars with substantial mass loss are relatively cool ( $T_{\text {eff }} \lesssim 3000 \mathrm{~K}$ ) objects (spectral types $\sim \mathrm{M} 6$ or later), whereas any stellar companions and/or accretion disks around them are likely significantly hotter $\left(T_{\text {eff }} \gtrsim 6000 \mathrm{~K}\right)$. Observed and model spectra of AGB stars show that their fluxes die rapidly at wavelengths shortward of about $2800 \AA$; hence significantly favorable secondary/accretion disk-to-primary flux contrast ratios ( $\gtrsim 10)$ are reached in the NUV window of Galaxy Evolution Explorer (GALEX) for companions of spectral type hotter than about $\mathrm{G} 0$ $\left(T_{\text {eff }}=6000 \mathrm{~K}\right)$ and luminosity $L \gtrsim 1 L_{\odot}$. We used UV imaging with GALEX to carry out a pilot search for binary companions in AGB stars (Sahai et al. 2008, hereafter Setal08). In this survey, we detected all of our 21 targets in the GALEX NUV filter with signal-to-noise ratios $(\mathrm{S} / \mathrm{N}) \gtrsim 20$; nine sources were detected in the FUV band with $\mathrm{S} / \mathrm{N} \gtrsim 8 \sigma$. In this Letter, we report the discovery of UV emission from the AGB star, Y Gem, from archival GALEX data.

\section{OBSERVATIONS AND RESULTS}

Y Gem was in the field of view of the GALEX Medium Imaging Survey and All-Sky Imaging Survey during three epochs from 2006 January to 2008 January that are roughly 

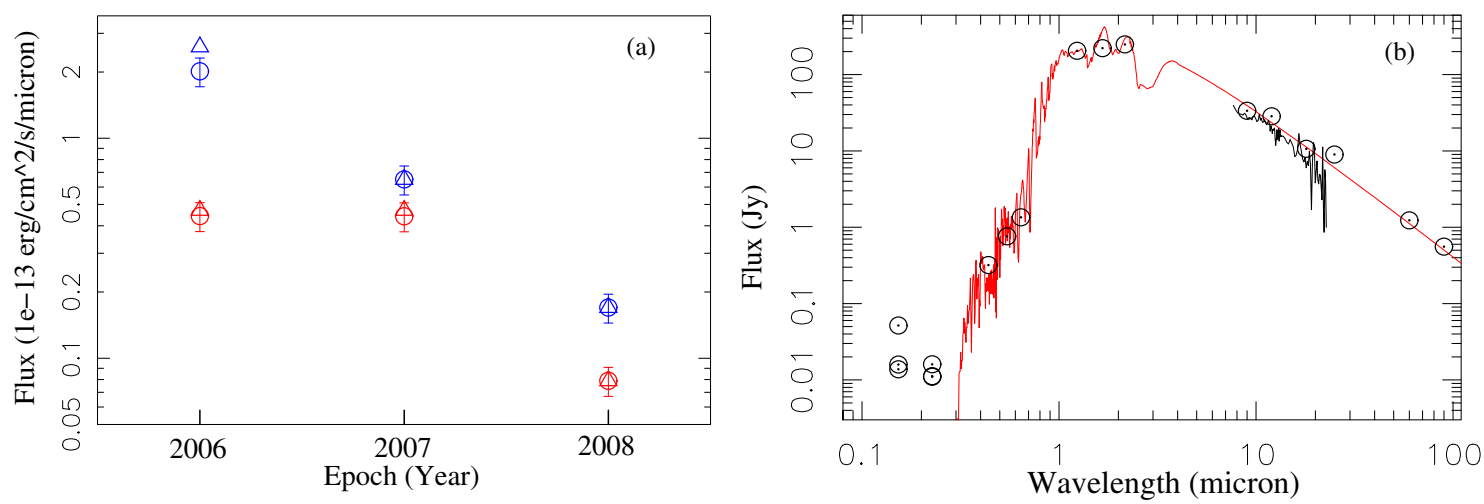

Figure 1. (a) The variable UV emission from Y Gem. Both uncorrected values (circles) and corrected ones (for nonlinearity, triangles) of the FUV (blue symbols) and NUV (red symbols) fluxes vs. epoch are shown (the $0.15 \mathrm{mag}$ error bars on the uncorrected values are the maximum zero-point error that the GALEX images have had since the early mission data were released). (b) The observed SED: photometry from GALEX (UV), USNO-B.1 and GSC2.3.2 ( $B, V, R), 2 \mathrm{MASS}(J, H, K$ ), AKARI-MID $(9,18 \mu \mathrm{m})$, AKARI-FIS $(90 \mu \mathrm{m})$, and IRAS $(12,25,60 \mu \mathrm{m})$ is shown as black circles and the IRAS/LRS spectrum as a black curve. A low-gravity $T_{\text {eff }}=2800 \mathrm{~K}$ model atmosphere spectrum from Lejeune et al. (1997) corresponding to Y Gem's M 8III spectral type (red curve) has been fitted to the SED.

Table 1

GALEX UV Photometry of Y Gem \& Mira

\begin{tabular}{|c|c|c|c|c|c|c|c|c|c|}
\hline Source & $\begin{array}{c}\text { Epoch } \\
\text { (yyyy-mm) }\end{array}$ & $\begin{array}{l}\text { Time } \\
(\mathrm{s})\end{array}$ & FUV & NUV & (cps) ${ } \mathrm{FUV}_{c}$ & $\mathrm{NUV}_{c}$ & $\begin{array}{l}\mathrm{FUV}_{c} \\
\left(10^{-13}\right.\end{array}$ & $\begin{array}{l}\mathrm{NUV}_{c} \\
\left.\mathrm{~m}^{-2} \AA^{-1}\right)\end{array}$ & $(\mathrm{FUV} / \mathrm{NUV})_{c}$ \\
\hline Y Gem & 2006-01 & 114 & 143.4 & 214.3 & 185 & 227 & 2.6 & 0.47 & 5.53 \\
\hline Y Gem & $2007-02$ & 109 & 46.5 & 216.5 & 46.5 & 230 & 0.65 & 0.47 & 1.38 \\
\hline Y Gem & 2008-01 & 1531 & 12.4 & 38.1 & 12.4 & 38.1 & 0.17 & 0.079 & 2.15 \\
\hline Mira & 2003-11 & 109 & 28.1 & 731.4 & 28.1 & 1032 & 0.39 & 2.13 & 0.18 \\
\hline Mira & 2006-11 & 11324 & 3.56 & 49.2 & 3.56 & 49.2 & 0.05 & 0.10 & 0.5 \\
\hline
\end{tabular}

spaced apart by 1 year. We discovered Y Gem's remarkable UV emission serendipitously while examining the GALEX archive for UV-emitting AGB stars. Y Gem, otherwise an unremarkable, semi-regular pulsating star of spectral type M8, turns out to be the brightest UV-emitting object among our sample of about one hundred M4-M8 stars with detected FUV emission (R. Sahai et al. 2012, in preparation). Following this discovery, we searched for CO $J=2-1$ line emission from Y Gem on 2011 February 11 and 15, using the ARO 10 m Submillimeter Telescope (SMT) on Mt. Graham, AZ, with the $1 \mathrm{~mm}$ dualpolarization receiver employing ALMA Band 6 SBS mixers. Typical system temperatures were $170-250 \mathrm{~K}$. The beam size was $\theta_{b}=32^{\prime \prime}$, and pointing accuracy is estimated to be about $\pm 4^{\prime \prime}$. Observations were conducted in beam-switching mode with a $\pm 3^{\prime}$ subreflector throw.

Y Gem was detected in both UV bands in each epoch, but its ultraviolet flux decreased dramatically from 2006 to 2008 (Table 1) - i.e., by a factor 12 (5.6) in the FUV (NUV) (Figure 1(a)). A model atmosphere corresponding to an M 8III spectral type from Lejeune et al. (1997) fitted to the spectral energy distribution (SED) from blue to the far-infrared shows that the UV flux densities are much larger than expected from Y Gem's photosphere (Figure 1(b)). Several of the flux values in 2006 and 2007 are large enough that nonlinearity corrections, as estimated from Morrissey et al. (2007), were significant and were used to correct the former; the corrected values are also provided in Table 1. An examination of the time-tagged photon lists (averaged over $10 \mathrm{~s}$ bins) showed no significant variability of the UV flux densities within each epoch, i.e., over periods of about 110 s during Epochs 1 and 2, and 1531 s during Epoch 3 . The FUV flux appears to decrease much faster than the NUV, with the ratio of the FUV-to-NUV wavelength-specific fluxes, $R_{\mathrm{FUV} / \mathrm{NUV}}$, taking uncorrected (corrected) values of 5.53 (4.54), 1.38 (1.47), and 2.15 (2.15) in 2006, 2007, and 2008, respectively. Y Gem had an FUV flux of $16 \mathrm{mJy}$ in 2006, much above the range of fluxes, $\sim 0.01-0.1 \mathrm{mJy}$, observed in the $9 / 21$ sources in our pilot survey with FUV emission.

The value of $R_{\mathrm{FUV} / \mathrm{NUV}}$ can be used to estimate the equivalent blackbody temperature of the emitting region, $T_{\mathrm{bb}}$. Since it is difficult to assess the relative line and continuum contributions to the FUV and NUV fluxes, we follow Robinson et al.'s (2005) arguments for arbitrarily (but conservatively) assuming that half (all) of the FUV (NUV) flux is continuum emission. Thus, we find that in 2006, $T_{\mathrm{bb}}=(3-3.8) \times 10^{4} \mathrm{~K}$ (for the uncorrectedto-corrected range of $R_{\mathrm{FUV} / \mathrm{NUV}}$ values) when the UV flux was at its peak and $T_{\mathrm{bb}}=1.7 \times 10^{4} \mathrm{~K}$ when the UV flux was at its lowest. The physical mechanism generating the UV emission is extremely energetic, with an integrated luminosity of (2.4-4) $L_{\odot}$ at its peak in 2006.

We detected weak CO $J=2-1$ emission from Y Gem (Figure 2). The line profile appears centrally peaked, with a full width at zero intensity (FWZI) of $5 \mathrm{~km} \mathrm{~s}^{-1}$ and an integrated flux of $0.044 \mathrm{~K} \mathrm{kms}\left(1.36 \mathrm{Jy} \mathrm{km} \mathrm{s}^{-1}\right.$ assuming an unresolved source). The CO line-width (FWZI) in Y Gem is quite small, compared to typical values for dusty outflows in AGB stars, $>20 \mathrm{~km} \mathrm{~s}^{-1}$ (e.g., CO catalog by Loup et al. 1993). Although we cannot rule out the possibility that Y Gem's CO emission comes from an anomalously slow outflow, it is more likely that it arises in a large, orbiting reservoir of molecular material in this object. Jura \& Kahane (1999) argue that such narrow lines, also seen in a few other evolved stars (e.g., EU And), most likely arise from long-lived reservoirs of orbiting gas of size (100-1000) AU. The dpAGB star, AC Her, a known binary with a compact disk (Gielen et al. 2007), also shows a similarly narrow CO line (Alcolea \& Bujarrabal 1991).

We estimate a distance $D=580$ pc to Y Gem from its TMSS $K$-band magnitude $m_{K}=1.22$, following Kahane \& Jura (1994) who assume that late-M semi-regular stars have 


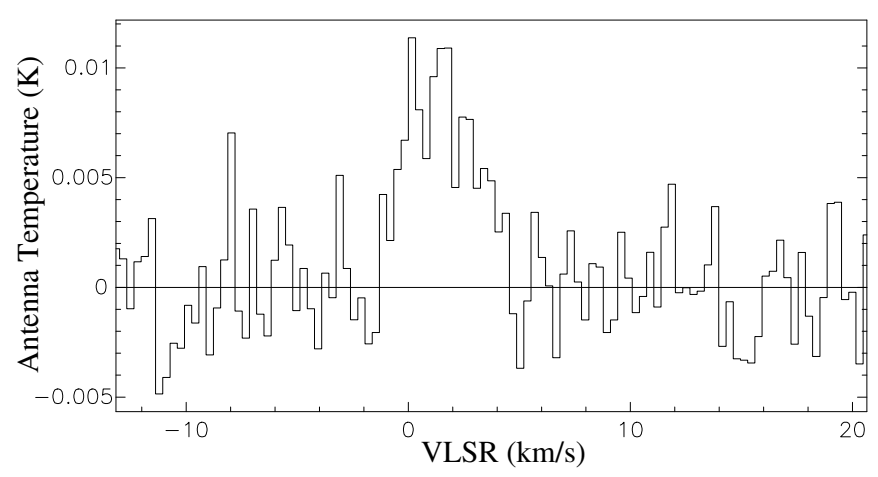

Figure 2. CO $J=2-1$ line emission from Y Gem, observed with the Arizona Radio Observatory's 10 m SMT millimeter-wave telescope. The temperature scale is measured as $T_{A}^{*}$, where the source's radiation temperature is defined as $T_{R}=T_{A}^{*} / \eta_{b}$, and $\eta_{b}(=0.74)$ is the main-beam efficiency.

absolute magnitudes $M_{K}=-7.6$. Assuming that the gas resides in a Keplerian disk around a star of $1 M_{\odot}$, the speed of $1.7 \mathrm{~km} \mathrm{~s}^{-1}$ (assumed to be half the FWHM of the CO line) indicates an orbital radius of $300 \mathrm{AU}$. At this distance from the star (with luminosity of $5800 L_{\odot}$ ), a blackbody grain would be at a temperature of $T_{d}=140 \mathrm{~K}$. We adopt this temperature for the circumstellar gas, following Jura \& Kahane's (1999) similar assumption for the disk in EU And. Assuming optically thin emission, the total gas mass is $M_{g}=$ $2 \times 10^{-5}\left(4 \times 10^{-4} / f_{\mathrm{CO}}\right) M_{\odot}$, where $f_{\mathrm{CO}}$ is the fractional COto- $\mathrm{H}_{2}$ abundance. Given this low gas mass, the fact that we can discern no dust emission in Y Gem's SED - the mid- and farinfrared fluxes are well fitted with photospheric emission from the M 8 primary (Figure 1(b)) - is not surprising. Assuming a typical circumstellar gas-to-dust ratio for AGB stars of 200, the expected 60 and $90 \mu \mathrm{m}$ fluxes from $140 \mathrm{~K}$ dust are 0.5 and $0.1 \mathrm{Jy}$, significantly smaller than the photospheric flux at these wavelengths.

\section{DISCUSSION}

Noting that the observed FUV fluxes in their sample were at least a factor $\sim 30$ higher than that can be produced by the continuum of the cool primary AGB star, Setal08 presented two plausible models for the FUV excesses-(1) the excess is due to the photosphere of a hot companion star, and (2) the excess is due to line and/or continuum emission associated with accretion activity and a disk around a companion star-the accretion shock may reside on (a) the disk or (b) the stellar surface of the companion (as, e.g., in T Tauri stars; Calvet \& Gullbring 1998). A third model is emission from a companion related to either (a) flare activity on an $\mathrm{M}$ dwarf (dM) companion or (b) coronal (magnetic) activity of a late-type (most likely $\sim$ F7-M4) mainsequence companion which has been spun up by wind accretion from the primary AGB star (Jeffries \& Stevens 1996; Soker \& Kastner 2002). A fourth model is variable chromospheric-type line emission from the AGB star as has been inferred in the C star, TW Hor, based on its IUE spectra (Querci \& Querci 1985). A fifth model follows from the proposal by Soker \& Kastner (2003) of the possibility of long-duration flares in single AGB stars due to magnetic reconnection events leading to X-ray emission. The only AGB star with an observed X-ray transient is Mira A which is in a (symbiotic) binary system (Karovska et al. 2005); and a sensitive search for X-rays in two (apparently) single AGB stars yielded upper limits at levels less than (1-10)\% of expectations (Kastner \& Soker 2004). Hence, it is not clear whether magnetic reconnection events in single AGB stars can produce X-ray flares; however, it would be worth investigating whether the latter can power the kind of strong, variable UV emission seen in Y Gem.

We think model 3a is unlikely. Flares on $\mathrm{M}$ dwarfs have been seen in the UV using GALEX (Welsh et al. 2007); however the decay timescales for the flare emission tend to be rather short-e.g., Figure 2 of Welsh et al. (2007) shows that most M-dwarf flare sources decay to half their peak flux in $\lesssim 100 \mathrm{~s}$. In contrast, Y Gem's flux showed no significant variations during any of the three epochs. Furthermore, dM flares are significantly less luminous in the NUV and FUV than Y Gem: e.g., scaled to Y Gem's estimated distance of $580 \mathrm{pc}$ (see below), the peak NUV flux of the most luminous flare object (J023955.52 - 072855.4) out of a total of $49 \mathrm{dM}$ stars in Welsh et al's survey (who only report NUV fluxes) is roughly a factor 35 fainter than Y Gem's peak (corrected) NUV flux, and the giant flare in the dMe star GJ 3685A is a factor 80 fainter in the FUV than Y Gem (Robinson et al. 2005).

Model 3b was proposed by Soker \& Kastner (2002) in order to explain the X-ray emission observed from the central binary stars of PNs and one can imagine extrapolating it to predict variable chromospheric UV emission from the spun-up companion as well. However, we do not think models $3 \mathrm{~b}$ and 4 are plausible models for Y Gem because for chromospheric emission, the ratio of the FUV-to-NUV fluxes (in mJy units) as measured in the GALEX bands, $r_{\mathrm{FUV} / \mathrm{NUV}}$, is much smaller than unity (about $0.1-0.05$ in TW Hor, and about 0.1 in the red supergiant $\alpha$ Ori), and results from the presence of strong emission lines in the NUV band. The large value of $r_{\mathrm{FUV} / \mathrm{NUV}}$ in Y Gem cannot be a result of dust attenuation because this would either not significantly change the FUV-NUV color or would redden the UV spectrum.

The highly variable UV flux in Y Gem supports model 2 above for this source; in this scenario, the UV emission from Y Gem may indicate episodic accretion (a) onto the disk, from matter ejected by the AGB star (e.g., Mastrodemos \& Morris 1998), or (b) onto the companion from the inner disk region. A likely example of the type 2 a mechanism is provided by Mira, in which the compact companion (separation $\sim 60 \mathrm{AU}$ ) accretes matter in a disk from the primary's wind. Mira shows variable NUV and FUV line emission: UV lines observed with IUE during 1979-95 faded by a factor $>20$ by 1999-2001, and then started increasing back to their original levels by 2004 (Wood \& Karovska 2006); the continuum was also variable. These authors concluded that the UV variability results from variations in the accretion rate (Mira A contributes to the UV flux only at wavelengths $\gtrsim 2600 \AA$ and only near its maximum). Mira was observed with GALEX on two different epochs separated by 3 years, and we find that its FUV and NUV fluxes changed dramatically over this period as well (Table 1).

Noting that Y Gem has never been classified as a symbiotic star, it is likely that its companion is a main-sequence star. If the emission is of type $2 \mathrm{~b}$, this inference is supported by our estimate of the emitting region's area, $A_{\text {emm }}$, which is $3.3 \times 10^{-3}$ and $(2.1-3.3) \times 10^{-3}$ times the total surface area of a Sunsized companion in 2008 and 2006, respectively (based on its UV flux and inferred blackbody temperature) - it exceeds the surface area of a white dwarf by at least a factor five. We note that, in published (magnetospheric) accretion-shock models for T Tauri stars (Gullbring et al. 2000; Calvet \& Gullbring 1998), even the largest values of the energy flux of the accretion flow, 
$\mathcal{F} \sim 10^{12} \mathrm{erg} \mathrm{cm}^{-2} \mathrm{~s}^{-1}$ (which determines the spectral shape of the UV emission), cannot produce the lowest value of $R_{\mathrm{FUV} / \mathrm{NUV}}$ seen in Y Gem. Thus, if Y Gem's UV emission is due to a magnetospheric accretion shock on the companion, then $\mathcal{F}$, and thus the mass accretion rate-typically $(0.1-$ few $) \times 10^{-7} M_{\odot}$ in these models-has to be significantly higher than in T Tauri stars.

Y Gem's relatively blue color over the wavelength region spanning the GALEX NUV-FUV bands is very similar to that of the carbon star V Hya, in which Setal08 found $r_{\mathrm{FUV} / \mathrm{NUV}} \sim 1.1$ in two separate epochs. V Hya is probably the best example to date of an evolved star with an active, collimated outflow, dense equatorially flattened structures possibly related to a central accretion disk, and an inferred binary companion and/or accretion disk from UV excesses (Setal08)

Y Gem appears to be one of the most prominent examples of a late-AGB star with a (inferred) mass-accreting binary companion, but is not alone, given the results of our survey (Setal08). However, being the brightest, it is the best one to begin investigating in order to ultimately determine whether the kind of binary interaction observed in Mira is generalized among UV-variable AGB stars-such binary interactions are widely believed to be the key to the formation of aspherical structure observed so commonly in pAGB objects. The aspherical structure has been characterized via specific descriptors in a comprehensive morphological classification system, developed for PPNs and extended to young PNs (Sahai et al. 2007, 2011). The descriptors primarily cover the shapes of the extended nebula and the central region. Two major classes of pAGB objects show equatorially flattened central structures. The first class of pAGB objects consists of PPNs and PNs; a large fraction of these harbor overdense, dusty equatorial waists that are quite large, e.g., in several PPNs, the waists can be seen to display sharp outer (radial) edges, with radii typically $\gtrsim 1000$ AU (Sahai et al. 2007). In the second class, namely, dpAGB objects, there is strong evidence for medium-sized ( $\sim 50 \mathrm{AU})$ disks (e.g., van Winckel et al. 2008) and little or no nebulosity. The relatively small CO line-width in Y Gem, implying the presence of a gravitationally bound structure, rather than a typical AGB dusty outflow, suggests that it will most likely evolve into a dpAGB object, rather than a PPN.

Ultraviolet spectroscopic observations of $\mathrm{Y}$ Gem using Hubble Space Telescope's Cosmic Origins Spectrograph are needed to probe the nature of the UV emission-i.e., if it is composed solely of emission lines arising in an accretion disk, or if there is a substantial continuum contribution (e.g., from the disk itself or the companion's photosphere). Any emission lines detected can provide constraints on the physical parameters of the accretion disk such as the (electron) density, emission measure, and thus the emitting volume (e.g., Reimers \& Cassatella 1985). Multi-epoch observations (either at UV or radio wavelengths) can constrain the size of the emitting region depending on the timescale of the variability. In a type $2 b$ mechanism, one might expect gyroresonance or gyrosynchrotron emission at radio wavelengths from Y Gem-e.g., Skinner \& Brown (1994) conclude that a likely model for the $2-3.6 \mathrm{~cm}$ emission in the pre-main-sequence star, $\mathrm{T}$ Tau $\mathrm{S}$, is non-thermal gyrosynchrotron emission arising in a scaled-up solar-like flare. Observations of high- $J$ CO lines such as $J=6-5,7-6,8-7$, and 9-8 with the Herschel Space Observatory are necessary to determine the temperature of the molecular gas in the disk.

We thank the staff of the Arizona Radio Observatory for granting us observing time. We thank Noam Soker and Joel Kastner for their valuable comments on an earlier version of this Letter. R.S.'s contribution to the research described here was carried out at the Jet Propulsion Laboratory, California Institute of Technology, under a contract with NASA. Financial support was provided by NASA through a Long Term Space Astrophysics and GALEX GO award.

\section{REFERENCES}

Alcolea, J., \& Bujarrabal, V. 1991, A\&A, 245, 499

Balick, B., \& Frank, A. 2002, ARA\&A, 40, 439

Bodenheimer, P., Burkert, A., Klein, R. I., \& Boss, A. P. 2000, in Protostars and Planets IV, ed. V. Mannings, A. P. Boss, \& S. S. Russell (Tucson, AZ: Univ. Arizona Press), 675

Bond, H. E. 2000, in ASP Conf. Ser. 199, Asymmetrical Planetary Nebulae II: From Origins to Microstructures, ed. J. H. Kastner, N. Soker, \& S. Rappaport (San Francisco, CA: ASP), 115

Calvet, N., \& Gullbring, E. 1998, ApJ, 509, 802

De Marco, O. 2009, PASP, 121, 316

Gielen, C., van Winckel, H., Waters, L. B. F. M., Min, M., \& Dominik, C. 2007, A\&A, 475, 629

Gullbring, E., Calvet, N., Muzerolle, J., \& Hartmann, L. 2000, ApJ, 544, 927

Hrivnak, B. J., Lu, W., Bohlender, D., et al. 2011, ApJ, 734, 25

Jeffries, R. D., \& Stevens, I. R. 1996, MNRAS, 279, 180

Jura, M., \& Kahane, C. 1999, ApJ, 521, 302

Kahane, C., \& Jura, M. 1994, A\&A, 290, 183

Karovska, M., Schlegel, E., Hack, W., Raymond, J. C., \& Wood, B. E. 2005, ApJL, 623, L137

Kastner, J. H., \& Soker, N. 2004, ApJ, 608, 978

Lejeune, T., Cuisinier, F., \& Buser, R. 1997, A\&AS, 125, 229

Loup, C., Forveille, T., Omont, A., \& Paul, J. F. 1993, A\&AS, 99, 291

Mastrodemos, M., \& Morris, M. 1998, ApJ, 497, 303

Miszalski, B., Jones, D., Rodríguez-Gil, P., et al. 2011, A\&A, 531, A158

Morrissey, P., Schiminovich, D., Barlow, T. A., et al. 2007, ApJ, 619, L7

Querci, M., \& Querci, F. 1985, A\&A, 147, 121

Reimers, D., \& Cassatella, A. 1985, ApJ, 297, 275

Robinson, R. D., Wheatley, J. M., Welsh, B. Y., et al. 2005, ApJ, 633, 447

Sahai, R., Findeisen, K., Gil de Paz, A., \& Sáanchez Contreras, C. 2008, ApJ, 689, 1274

Sahai, R., Morris, M., Sánchez Contreras, C., \& Claussen, M. 2007, AJ, 134, 2200

Sahai, R., Morris, M. R., \& Villar, G. G. 2011, AJ, 141, 134

Sahai, R., \& Trauger, J. T. 1998, AJ, 116, 1357

Schwarz, H. E., Corradi, R. L. M., \& Melnick, J. 1992, A\&AS, 96, 23

Skinner, S. L., \& Brown, A. 1994, AJ, 107, 1461

Soker, N. 1998, ApJ, 496, 833

Soker, N., \& Kastner, J. H. 2002, ApJ, 570, 245

Soker, N., \& Kastner, J. H. 2003, ApJ, 592, 498

Van Winckel, H., Deroo, P., Gielen, C., et al. 2008, in AIP Conf. Proc. 1001, IXth Torino Workshop on Evolution and Nucleosynthesis in AGB Stars and the IInd Perugia Workshop on Nuclear Astrophysics (Melville, NY: AIP), 349

Van Winckel, H., Lloyd, E. T., Briquet, M., et al. 2009, A\&A, 505, 1221

Welsh, B. Y., Wheatley, J. M., Seibert, M., et al. 2007, ApJS, 173, 673

Wood, B. E., \& Karovska, M. 2006, ApJ, 649, 410 